\title{
GSM BASED AUTOMATIC LPG ORDERING SYSTEM WITH LEAKAGE ALERT
}

\author{
Abid Khan', Neju K. Prince ${ }^{2}$, Shailendra Kumar Dewangan ${ }^{3}$, Praveen Singh Rathore ${ }^{4}$ \\ ${ }^{l}$ Department of Electronics \& Telecommunication Engg, Christian College of Engg \& Technology, Bhilai, \\ Chhattisgarh \\ ${ }^{2}$ Department of Electronics \& Telecommunication Engg, Christian College of Engg \& Technology, Bhilai, \\ Chhattisgarh \\ ${ }^{3}$ Department of Electronics \& Instrumentation Engg, Chhatrapati Shivaji Institute of Technology, Durg, Chhattisgarh \\ ${ }^{4}$ Department of Electronics \& Telecommunication Engg, Christian College of Engg \& Technology, Bhilai, \\ Chhattisgarh
}

\begin{abstract}
Now days we all are very busy in our daily life and it is difficult to know the status of LPG gas cylinder. If LPG is going to finish without informing us it can create very difficult condition for cooking etc. Our proposed design can help us to avoid such kind of problem in our daily life. Our design is based on microcontroller, it can track LPG emptiness all the time if LPG is very close to finish or at empty level then it can alert us by sending SMS to owner \& it can also transfer message to LPG Agency for ordering the LPG cylinder. In addition it can provide safety also by using sensor it can detect LPG leakage \& start alarm.
\end{abstract}

Keywords: LPG, GSM, SMS, LCD, GAS Sensor MQ-6, Microcontroller

\section{INTRODUCTION}

The main task of proposed design is to measure the gas present in the cylinder going below the fixed level .In our proposed design we are not going to do any arrangement on cylinder because it is dangerous. We use knob position of gas stove. We detect consumption of gas according time. It is safe \& easy to implement .No need to go at cylinder side that provides safety also. We measure consummation of gas according to time. If gas reducing particular level. GSM transmit booking message for a new cylinder and the house owner (consumer) can receives the message about the same time. The alert mechanism in the proposed system includes an LED indication, buzzer and an SMS sent to the stored numbers with the help of GSM. [1] The Other main objective to prevent damage or explosion of LPG by using MQ 5 sensor. It can detect gas leakage and alert us by buzzer using Beep sound and send an alert SMS using GSM module.

\section{SYSTEM ARCHITECTURE}

Hardware framework for proposed system is shown in Fig 1. System Contains Microcontroller, LCD Display, LED, Buzzer and GSM Modem. All Devices Controlled By Microcontroller Unit. When Gas inside the cylinder goes below threshold limit it sends SMS alert to the user as well as gas agency using GSM module. LCD unit continuously display level of gas inside cylinder. It Display the Quantity of Gas Inside Cylinder.

When gas inside cylinder less than particular limit then microcontroller immediately send a booking message to gas agency \& at same time send alert message to user. The message will be displayed on LCD.

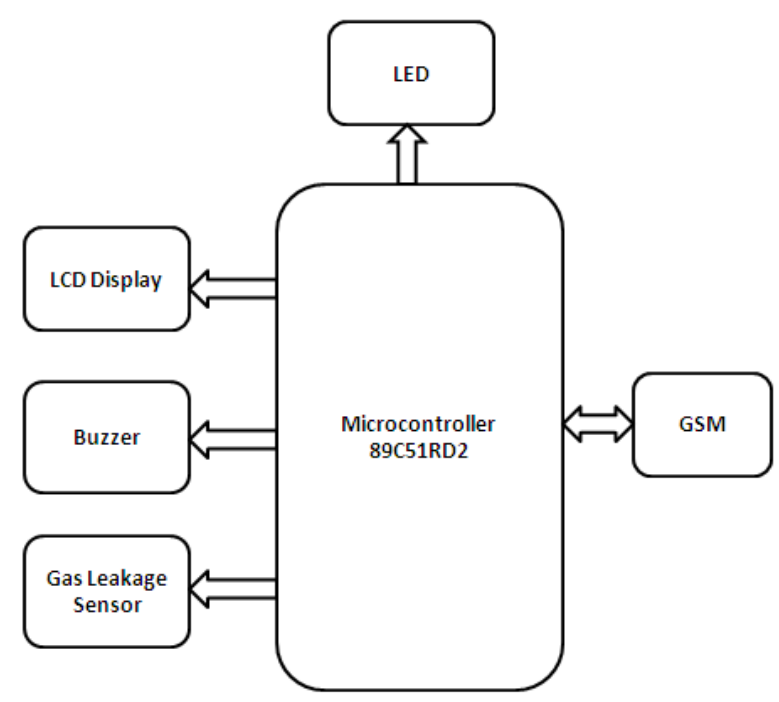

Fig. 1 - Framework for Proposed System

Proposed design can provide safety by using MQ5 gas sensor which can detect the gas leakage and gives signal to the microcontroller. It activates to buzzer \& using GSM module it can send warning message to the user. Proposed design able to receive message from gas agency it can display message received by gas agency when booking is confirmed. System flow diagram is shown below in Fig. 2.

\subsection{Microcontroller}

- A smaller computer

- On-chip RAM, ROM, I/O ports. 
Example 89C51RD2 Microcontroller perfectly fits many uses, from automotive industries and controlling home appliances to industrial, instruments, remote sensors, electrical door locks and safety devices. Low cost, low consumption, easy handling and flexibility make 89C51RD2 Microcontroller applicable.

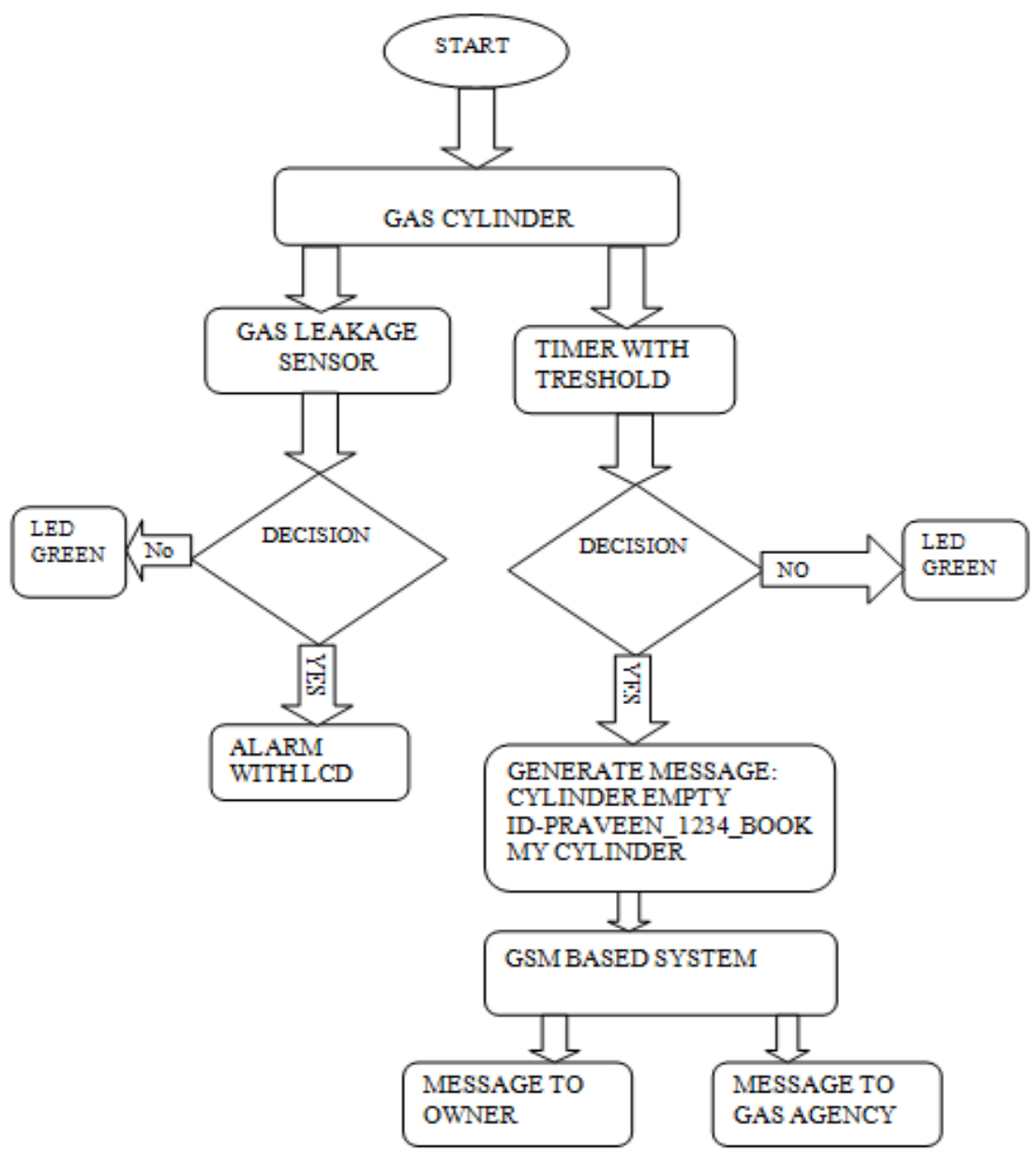

Fig. 2 - Block Diagram of Proposed System

\subsection{Buzzer}

Piezoelectric buzzers are used as cheap, dependable device to generate an alarm tone in electronic circuits. [3] Piezo buzzers are used for making beeps, tones and alerts.

\subsection{Gas Sensor (MQ-6)}

MQ6 is a semiconductor type gas sensor which detects the gas leakage. The sensitive material of MQ-6 is tin dioxide (SnO2). It has very low conductivity in clean air. This Gas sensor not only has sensitivity to propane and butane but also to other natural gases, low sensitivity to cigarette smoke.

\subsection{GSM Module}

SIM300 can be integrated with a wide range of applications. SIM300 is a Tri-band GSM/GPRS engine that works on frequencies EGSM $900 \mathrm{MHz}$, DCS $1800 \mathrm{MHz}$ and PCS1900 MHz SIM300 provides GPRS multi-slot class 10 capabilities and support the GPRS coding schemes CS-1, CS-2, CS-3 and CS-4. With a tiny configuration of $40 \mathrm{~mm} \times 33 \mathrm{~mm} \times 2.85$ $\mathrm{mm}$, SIM300 can fit almost all the space requirement in our
application.GSM modules need extensively on the market mainly are standard GSM modules

\section{RESULTS AND DISCUSSIONS}

The Different knob positions are shown in Fig. 3 (a), (b) \& (c). Consumption of gas depends on various knob positions. Consumption of gas at 0,1 \& 2 level is different. Consumption of gas can be calculated by mathematical formula for different knob position which use to detect gas level at cylinder.

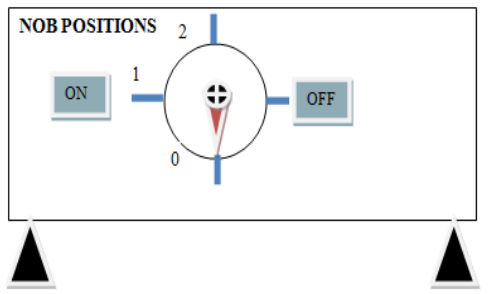

Fig. 3(a) - Knob at Zero Position 


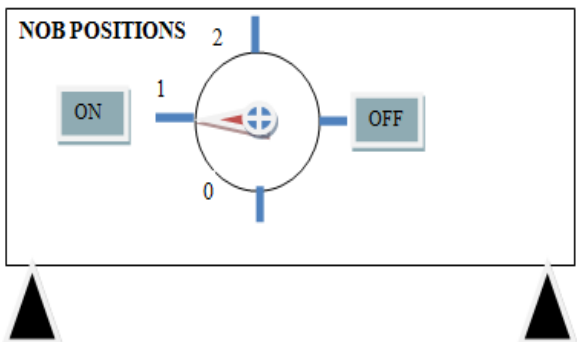

Fig. 3(b) - Knob at First Position

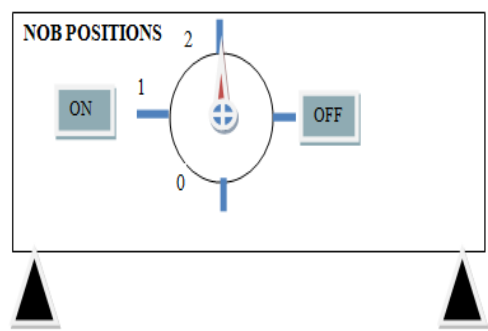

Fig. 3(c) - Knob at Second Position

\section{CONCLUSIONS}

The development process of this work include microcontroller for timer, GSM module for SMS, gas leakage sensor, alarm system and LED, LCD displays. It is completely integrated so that once it is implemented then it is possible to monitor gas leakage anytime. It has Real-time capability and low cost and able to forms a real-time accurate, effective system. The advantage of this automated detection and alerting system over the manual method is that it offers quick response time, easy to use, simplicity of design and Easy implementation.

\section{REFERENCES}

1. K. Padmaj Priya, M. Surekha, R. Preethi, T. Devika \& N. Dhivya, "Smart Gas Cylinder Using Embedded System," International Journal of Innovative Research In Electrical, Electronics, Instrumentation \& Control Engineering, Vol. 2, Issue 2, February 2014.

2. Ramya \& B. Palaniappan, "Embedded System for Hazardous Gas Detection \& Alerting”, International Journal of Distributed and Parallel Systems, Vol. 3, No. 3, May 2012.

3. C. Yogeesh, P. Ashwini \& B. P. Shruthi, "Automated Unified System for LPG Refill Booking \& Leakage Detection: A Pervasive Approach", International Journal of Advanced Technology \& Engineering Research.

4. Sagar Shinde, S. B. Patil \& Dr. A. J. Patil, "Development of Movable Gas Tanker Leakage Detection using Wireless Sensor Network based on Embedded System", International Journal of Engineering, Research \& Applications, Vol. 2, Issue 6, pp. 1180-1183, December 2012.
5. Mahalingam, R. T. Naayagi \& N. E. Mastorakis, "Design and Implementation of an Economic Gas Leakage Detector", Recent Researches in Applications of Electrical and Computer Engineering.

6. S. Rajitha \& T. Swapna, "Security Alert System Using GSM for Gas Leakage”, International Journal of VLSI and Embedded Systems,

7. J. Sunitha \& D. Sushmitha, "Embedded Control System for LPG Leakage Detection \& Prevention", International Conference on Computing and Control Engineering, April, 2012. 